\title{
Varied Presentation of Unusual Soft Tissue Lesions- A Case Series
}

\author{
Binjul Juneja, Mohd Talha, Saquib Alam and Kafil Akhtar* \\ Departments of Pathology, Aligarh Muslim University, India
}

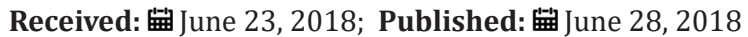

*Corresponding author: Kafil Akhtar, Professor, Department of Pathology, Jawaharlal Nehru Medical College, Aligarh Muslim University, Aligarh(U.P), India

\begin{abstract}
Malignant chest wall tumors are broadly classified into eight main diagnostic categories: muscular, vascular, fibrous and fibrohistiocytic, peripheral nerve, osseous and cartilaginous, adipose, hematologic and cutaneous. Some other malignant chest wall tumors that do not fit well in any of such category are synovial sarcoma and ewing's sarcoma. Sarcomas of soft tissues, particularly those from the deep sites of the extremities raise a problem of diagnosis and treatment. Hemangiopericytoma (HPC) is a rare vascular tumor, and is most controversial, because earlier it was thought to represent a neoplasm of the pericytes of Zimmerman. Histiocytic sarcomas, including malignant fibrous histiocytoma (MFH), represent a group of neoplasms with an unpredictable course and for which treatment varies widely. Pleomorphic malignant fibrous histiocytoma (MFH) which is also known as undifferentiated high-grade pleomorphic sarcoma according to the latest World Health Organization classification is a diagnosis of exclusion. Myxoid liposarcoma (LS) is the most common subtype of liposarcoma and occurs predominantly in the extremities. Different cytogenetic features and their underlying molecular alterations define distinct entities among LS. Myxoid LS has a strong and specific association of the (12;16). Inflammatory fibrosarcoma, commonly referred to as inflammatory myofibroblastic tumor (IMT) has become as part of a spectrum of inflammatory myofibroblastic proliferation. It is potentially locally aggressive tumor of the mesentery of children and young adults. Immunohistochemistry plays an important role to distinguish different types of soft tissue tumors with similar morphology.
\end{abstract}

Keywords: Hemangiopericytoma; Pleomorphic malignant fibrous histiocytoma; Myxoid liposarcoma; Inflammatory fibrosarcoma.

\section{Introduction}

Hemangiopericytoma behaves aggressively with a high rate of local recurrence and distant metastases [1]. Hemangiopericytomas also represent rare intracranial tumors that have a tendency to recur locally and have the unique characteristic of extracranial metastases [2]. It has two histologic forms: conventional HPC and lipomatous HPC. Both the forms show a sponge-like sinusoidal vasculature and staghorn-shaped blood vessels which are haphazardly bounded and surrounded by ovoid and short spindle shaped cells. Histologic identification of lipomatous HPC is readily achieved because of an HPC like appearance with the added finding of a lipomatous component. Clinical presentation of conventional HPC is nonspecific. Pain is a late symptom associated with an enlarging mass; though symptoms vary depending on the site of disease. Characteristically, HPC is a well-circumscribed, brown, spongioform lesion, surrounded by a pseudo-capsule, often with small satellite nodules separate from the main tumor mass, whereas synovial sarcoma is grossly cream-colored on gross examination [3]

Malignant fibrous histiocytoma presents with a rapid tumorous growth as the major symptom. The lower extremities are the most frequent anatomic site $( \pm 50 \%)$. This tumor may occur at any age but has a predilection for the $6^{\text {th }}$ and $7^{\text {th }}$ decades in males. The most important clinical prognostic features include site, depth, volume and number of muscles involved and the integrity of the neurovascular structures. The inflammatory component, mitotic index, cellular polymorphism and paraneoplastic syndromes are some of the other prognostic factors. Liposarcoma (LPS) is considered as one of the most common histologic subtypes of adult soft tissue sarcoma. Myxoid liposarcoma is a painless, slowly growing mass present for several months to several years. These tumors 
are encapsulated, non-infiltrating, nodular masses of varying size and usually septated. Myxoid liposarcoma of the extremities and trunk wall rarely show distant metastasis [4]. Few cases present with a painful nodule [5]. Inflammatory fibrosarcoma which is commonly referred to as inflammatory myofibroblastic tumor is a potentially locally aggressive myofibroblastic tumor that occurs predominantly in the mesentery of children and young adults. They are characterized as solitary, well-demarcated fibrous tumors with numerous inflammatory cells, mainly lymphoid or plasma cells along and may have associated reactive lymphadenopathy.

\section{Case Summary}

Case 1: Hemangiopericytoma: A 26-year-old female patient presented to the Surgical Clinic with complaints of pain and mass in the right anterior chestwall for two months. On physical examination, pulmonary auscultation showed decreased respiratory sounds on right side. A hard mass of approximately $10 \times 10 \mathrm{~cm}$ was palpated in the midline of right chest wall. Other physical examination findings with medical and familial history was non-contributory. The complete blood count and routine biochemical analysis were normal. On PA chest radiography, a mass of approximately $10 \times 10 \mathrm{~cm}$ was seen in the right lung with right pleural effusion. On thoracic computerized tomography, a heterogeneous mass with lobular contour localized in right mid lobe with continuity to the anterior chest wall without forming a costal destruction was observed. The mass was seen pushing forward the pectoral muscle without invading the breast tissue. An incisional biopsy was obtained from the mass and the histopathologic examination showed a malignant mesenchymal tumor, rich in vessels. Thereafter, an operation was planned for the patient and the vascular tumor mass disseminating to extrapleural space and the bottom tip of the sternum and the 4 th, 5 th, 6 th, and 7 th costae was excised.

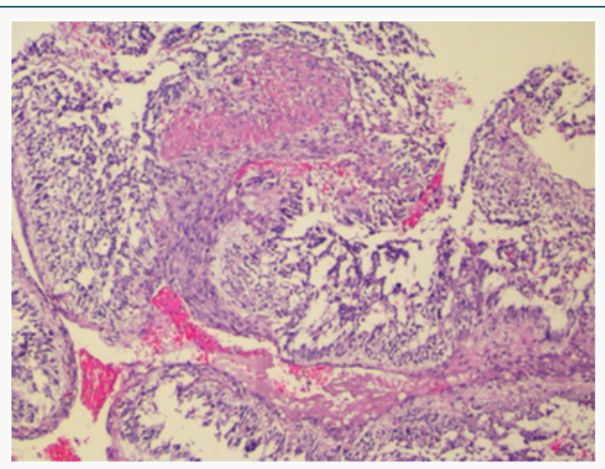

Figure 1: Hemangiopericytoma: Tissue section showed a high cellularity mass with uniform tumor cells with minimal pleomorphism, spindle to round to oval nuclei with vesicular to hyperchromatic chromatin and eosinophilic cytoplasm with indistinct cell borders and richly vascularized with staghorn-appearing vessels, with high mitotic activity. Hematoxylin and Eosin $x 40 X$.

The histopathologic examination showed a high cellularity mass with uniform tumor cells with minimal pleomorphism, spindle to round to oval nuclei with vesicular to hyperchromatic chromatin and eosinophilic cytoplasm with indistinct cell borders. The tumor was richly vascularized with staghorn-appearing vessels, with high mitotic activity (Figure 1). There was no evidence of tumor tissue infiltrating the bone and the cartilaginous tissues. On immunohistochemistry, the tumor cells were diffusely positive for CD34 and MIC-2 whereas staining for actin and EMA was negative. Our patient is doing well after 12 months of follow up.

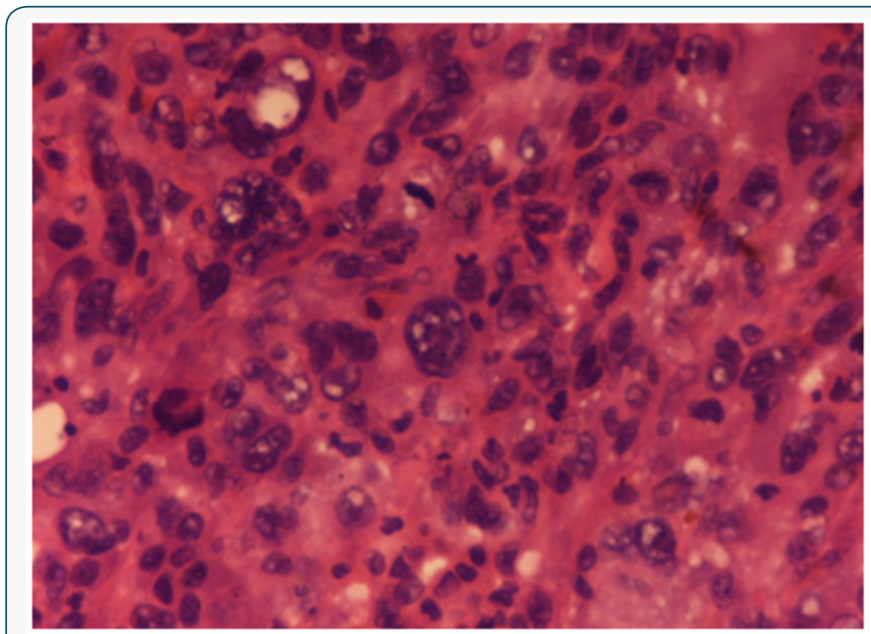

Figure 2: Malignant fibrous histiocy toma: Microscopically, the lesion showed marked architectural and cytologic pleomorphism with haphazardly arranged malignant plump to spindle shaped cells admixed with giant cells. Hematoxylin and Eosin $x$ 40X.

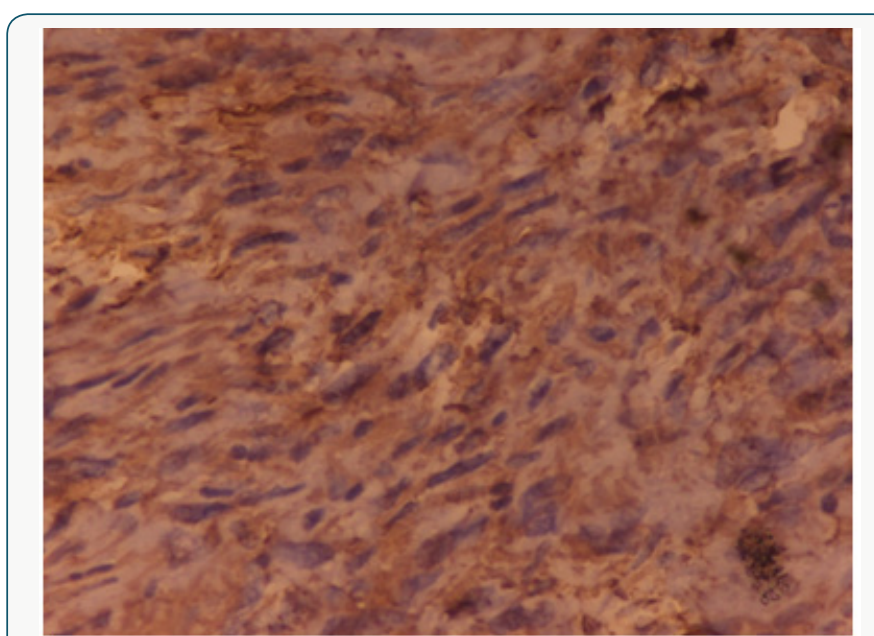

Figure 3: Malignant fibrous histiocytoma: The neoplastic cells were positive for vimentin. IHC Vimentin $\mathrm{x} 40 \mathrm{X}$.

Case 2: Pleomorphic Malignant Fibrous Histiocytoma (Mfh): A 63-year-old man presented with a thigh mass for 10 months. On local examination, the mass was $12 \mathrm{x} 9 \mathrm{~cm}$, firm in consistency with ill-defined margins. The specimen of emergent debridement was submitted for pathologic and bacteriologic examination. Microscopically, the lesion showed marked architectural and cytologic pleomorphism with haphazardly arranged malignant plump to spindle shaped cells admixed with giant cells (Figure 2). The neoplastic cells were positive for vimentin (Figure 3), but negative for all lineage-specific markers. The diagnosis of 
pleomorphic MFH was made. Thoracic computed tomography scan showed bilateral multiple pulmonary nodules. The patient died 1 month later.

Case 3: Myxoid Liposarcoma: A 25-year-old man presented to the Surgical Clinic with complaints of soft tissue mass in the left thigh for the last 3 months. On local examination, the mass was soft to firm in consistency, well circumscribed of $12 \times 10 \mathrm{~cm}$ size. Blood parameters were normal and there was no functional abnormality. He was operated upon with wide local excision. The excised mass was $10 \times 9.5 \mathrm{~cm}$, soft to gelatinous in consistency without necrosis or haemorrhage. Histopathological examination revealed a myxoid tumor comprising of small dark oval cells in a myxoid background. An extensive capillary network with typical lipoblasts were also seen with mitotic activity of 5 mitosis/ 10HPF (Figure 4). A diagnosis of myxoid liposarcoma was given. The initial surgery was accompanied with adjuvant chemotherapy and complementary radiotherapy. The patient was well after 12 months of follow up.

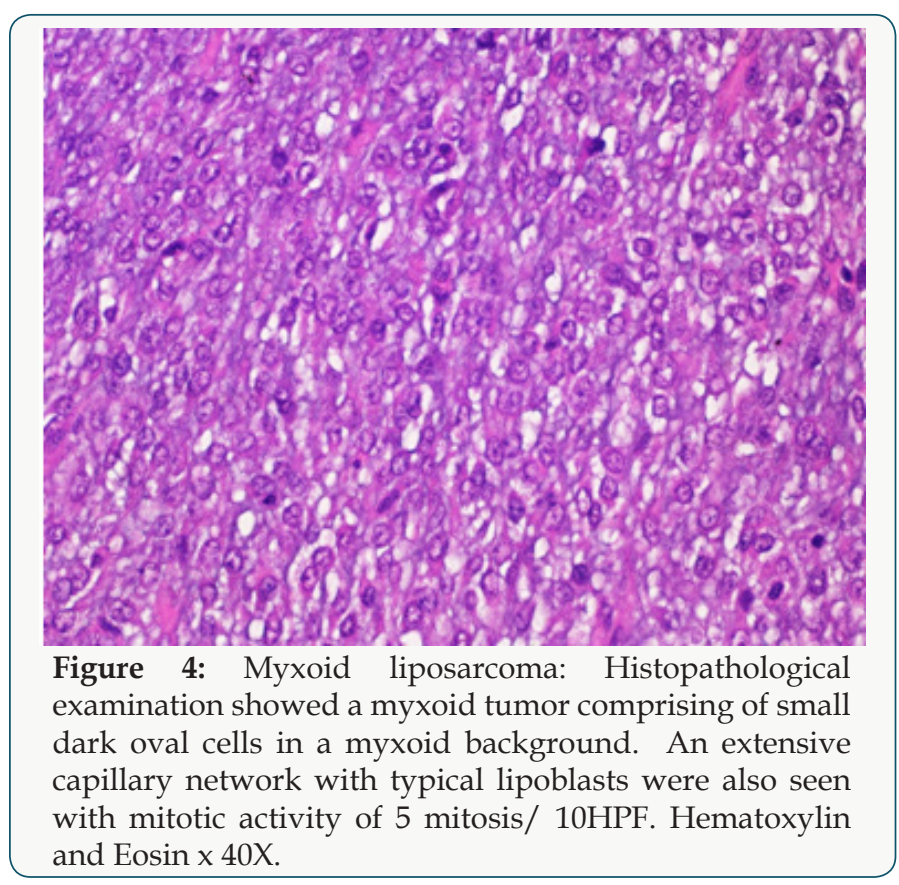

Case 4: Inflammatory Fibrosarcoma: A 42 years old male presented to the Surgery Out-patients Department with complaints of generalised severe pain in the abdomen, localized mainly in the right iliac fossa for 2 days. He was previously fit and healthy and started with generalised feeling of being unwell for four months with mild recurrent pain abdomen, bloating sensation, loss of weight over half a stone and loss of appetite. There was no history of bladder or bowel disturbance, fever, jaundice or vomiting. His pain was worse in the last 2 days associated with nausea. On clinical examination he appeared anxious and sweaty with mild dehydration. Abdominal examination showed tenderness and guarding in the right iliac fossa with the rest of the abdomen soft and there were no masses palpable. Rectal examination was unremarkable. A possible diagnosis of appendicitis was made.
Haematological investigations showed a raised white cell count of $17.800 / c c$ and biochemical investigations were within the normal limits. Chest X-Ray was normal with the abdomen plain film showing stones in the gall bladder and a few dilated small bowel loops. Urgent ultrasound scan of abdomen confirmed a small collection of fluid in the right iliac fossa and gallstones with no gas in the biliary tree. In view of the uncertain diagnosis a laparotomy was carried out. This revealed a large mass in the right iliac fossa at the Ileo-caecal junction. The distal ileum was adhered to caecum and adjacent mesentery with thick pus between the loops of small bowel. Right hemicolectomy and an end to side ileo-transverse anastomosis was performed. The postoperative recovery of the patient was uneventful. The histopathology was initially reported as highly cellular spindle cell tumour, with frequent mitotic activity with the most likely site of origin of tumour being bowel wall and spreading in to the surrounding areas including the mesentery. Tumour markers SMA, desmin and vimentin were positive there by indicating leiomyosarcoma. In view of the positive cytokeratin immunostaining, a second opinion was sought by pathologists. On further review, an "Inflammatory fibrosarcoma of the colon" was given due to the marked pleomophism and associated inflammatory cell infiltrate (Figure 5).

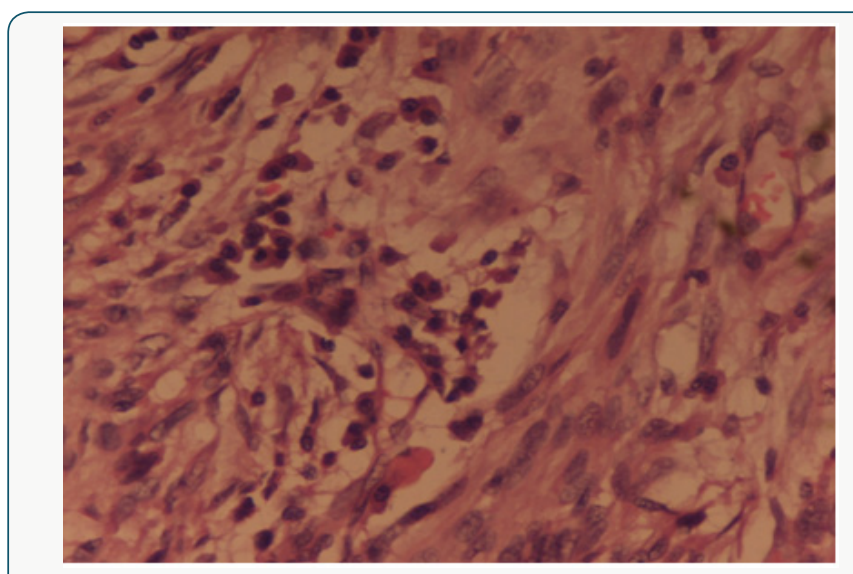

Figure 5: Inflammatory Fibrosarcoma: The histopathology revealed a cellular spindle cell tumour, with frequent mitotic activity with marked pleomophism and associated inflammatory cell infiltrate. Hematoxylin and Eosin x 40X.

\section{Discussion}

Hemangiopericytoma is a rare tumor of adult, found mainly in the fifth decade of life. It is most frequently mimicked by synovial sarcoma which occasionally presents as a pure hemangiopericytoma like lesion. These tumors can originate anywhere in the body where there are capillaries. The most common locations reported are the brain, lower extremities, pelvis and head \& neck. It originates in the pericytes, the cells normally arranged along specific types of blood vessels. It can be broadly classified as intracranial and extracranial. Extracranially, it can occur at any site throughout the body in soft tissues and bone. They have a grade 2 or 3 behavior and needs to be distinguished from benign meningiomas because 
of their high rate of recurrence (41\%) and metastases (12-20\%) [6]. They are highly cellular and mitotically active neoplasm that is rich in pericellular reticulin and stains with anti-type IV collagen. They can be distinguished from benign meningiomas by their hypercellularity, higher mitotic index and microscopically bulge into vascular lumens without bursting through the endothelium, exhibiting a characteristic well-developed "staghorn" branching vascular pattern [7]. They are painless masses and may not have any associated symptoms. They can remain undetected for long periods of time due to the fact that they originate in soft tissue except when intracranial it can cause neurological disturbances. Exhibiting a characteristic well-developed "staghorn" branching vascular pattern. Differential diagnosis includes synovial sarcoma (similar vascular pattern, characteristic translocation), mesenchymal chondrosarcoma (islands of mature cartilage; malignant chondrocytes present), fibrous histiocytoma (storiform pattern, fibrohistiocytic lesion) and solitary fibrous tumor (more prominent collagen, less prominent vessels). Hemangiopericytoma on immunohistochemistry stain positive for CD99, vimentin, CD 34 and negative for Factor VIII and CD31. In adults, complete surgical resection remains the mainstay of treatment. Malignant fibrous histiocytoma has more recently been classified as pleomorphic undifferentiated sarcoma (PUS). It is considered as the most common type of soft tissue sarcoma in adults and has an aggressive biological behaviour with poor prognosis $[8,9]$. Typically occurs in adults with a slight male predilection. The presentation is usually with a painless, enlarging and well circumscribed palpable mass. They are usually confined to the soft tissues having predilection for extremities but occasionally may arise in or from bone also (1-5\%). Some of its histological subtypes includes storiformpleomorphic, myxoid, myxofibrosarcoma, inflammatory, giant cell and angiomatoid. Pleomorphic malignant fibrous histiocytoma which is the most common subtype is considered a diagnosis of exclusion for sarcomas that cannot be more precisely categorized [10]. Imaging typically shows a well-circumscribed mass that is dark on T1-weighted images and bright on T2-weighted images. Histomorphology is characterized by high cellularity, marked nuclear pleomorphism accompanied by abundant mitotic activity including atypical mitoses and a spindle cell morphology. Necrosis is commonly present. Treatment consists of surgical excision and in almost all cases radiation eliminates the need for limb amputation.

Liposarcoma is a malignant tumor that arises from deep soft tissue fat and not from common lipomas. It accounts for up to $20 \%$ of all soft tissue sarcomas and commonly affects adult [11]. It can occur in almost any part of the body, commonly involving thigh and retroperitoneum. There are four subtypes, each having its unique characteristics: well-differentiated liposarcoma (most common subtype), myxoid/round cell liposarcoma, pleomorphic liposarcoma(rarest subtype) and dedifferentiated liposarcoma. Dedifferentiated liposarcoma and pleomorphic liposarcoma are considered as highly malignant [12]. Myxoid liposarcoma (MLS) is considered as a low grade tumor but the presence of areas of round cells more than $5 \%$ is associated with a worse prognosis [4]. MLS presents as a slow-growing, deep-seated tumor in the lower extremity of a relatively young adult. Specific chromosomal translocations have been discovered in MLS which consists of the fusion of the FUS and CHOP genes $[(\mathrm{t} 12 ; 16)(\mathrm{q} 13 ; \mathrm{p} 11)]$ in $90 \%$ of tumor [13]. Extremity myxoid liposarcomas have an unusually high predilection for extra-pulmonary metastases often deep soft tissue locations such as retroperitoneum or extrimities without any pulmonary metastases [14,15]. Imaging of the abdomen, retroperitoneum, and extrapleural chest should be performed for accurate staging and post-treatment follow-up of patients with myxoid liposarcoma. In all patients surgical management of the tumor is curative. It is radiosensitive as compared with other soft tissue sarcomas [16]. After treatment of the primary tumor, such patients should be followed with regular chest X-ray and abdominal/pelvic computed tomography (CT) scans.

Fibrosarcoma is a malignant neoplasm of mesenchymal origin in which histologically the predominant cells are fibroblasts that divide excessively without cellular control. Inflammatory fibrosarcoma is rare and was originally described in the lung by names such as pseudotumor, inflammatory pseudotumor and plasma cell granuloma. It is typically considered as a benign tumor with aggressive behavior (low-grade tumor) that can occur anywhere in the body. They are solitary, well-demarcated fibrous tumors and are characterized by storiform pattern of fibrous tissue along with of mixture of inflammatory cells such as plasma cells, lymphocytes and eosinophils as well as spindle cells without nuclear atypia [17]. These tumors may also have necrosis, hemorrhage, focal calcification and mitotic activity. Some of the histologic differential diagnosis includes: calcifying fibrous pseudotumor, inflammatory fibroid tumor and nodular fasciitis. Immunohistochemically, the tumor shows intense immunoreactivity for vimentin, muscle actin, and $\alpha$-smooth muscle actin but are negative for desmin and highmolecular-weight caldesmon. In addition, tumor cells are not labeled by antibodies against AE1/3 and CAM5.2. The prognosis is generally good on tumor removal, but rarely some tumors are known to metastasize.

\section{References}

1. Schiariti M, Goetz P, Hussein EM (2011) Hemangiopericytoma: long term outcome revisited clinical article. J Neurosurg 114(3): 747-755.

2. Fountas KN, Kapsalaki E, Kassam M (2006) Management of intracranial meningeal hemangiopericytomas: outcome and experience. Neurosurg Review 29(2): 145-153.

3. EspatJN, Jonathan J, LeungD (2002) Conventional hemangiopericytoma. Am Cancer Soc 95(8): 1746-1751.

4. Nishida Y, Tsukushi S, Nakashima H (2010) Clinicopathologic prognostic factors of pure myxoid liposarcoma of the extremities and trunk wall. Clin Ortho Res Res 468(11): 3041-3046.

5. Hsu YF, Chou YY, Cheng YH (2012) Spermatic cord myxoid liposarcoma presenting as an incarcerated inguinal hernia: report of a case and review of literatures. Hernia 16(6): 719-722. 
6. Melone AG, DElia A, Santoro F, Salvati M, Delfini R, et al. (2014) Intracranial hemangiopericytoma--our experience in 30 years: a series of 43 cases and review of the literature. World Neurosurg 81(4): 556562.

7. Gengler C, Guillou L (2006) Solitary fibrous tumour and haemangiopericytoma: evolution of a concept. Histopathol 48(1): 63-74

8. Al Agha OM, Igbokwe AA (2008) Malignant fibrous histiocytoma between the past and the present. Arch Pathol Lab Med 132(6): 10301035.

9. Nakayama R, Nemoto T, Takahashi H (2007) Gene expression analysis of soft tissue sarcomas: characterization of malignant fibrous histiocytoma. Mod Pathol 20(7): 749-759.

10. Matushansky I, Charytonowicz E, Mills J, Siddiqi S, Hricik T, et al. (2009) MFH classification: differentiating undifferentiated pleomorphic sarcoma in the $21^{\text {st }}$ Century. Anticancer Ther 9(8): 1135-1144.

11. Angelo P, Dei T (2000) Liposarcoma: New entities and evolving concepts. Ann Diagn Pathol 4(4): 252-266.

12. Fubio Li, Runhui T, Changjiu Y (2013) Liposarcoma of the spermatic cord mimicking a left inguinal hernia: a case report and literature review.
World J Surg Oncol 11: 18-19.

13. Suzan ET, Harald JH, Robert JVG (2007) Clinicopathologic prognostic factors in myxoid liposarcoma: a retrospective study of 49 patients with long term follow up. Ann Surg Oncol 14(1): 222-229.

14. Enzinger FM, Weiss SM (2014) Liposarcoma. In: Enzinger FM, Weiss SM, Soft Tissue Tumors. ( $6^{\text {th }}$ edn), Mosby, St. Louis MO, USA, pp: 641 693.

15. Estourgie SH, Nielsen GP, Ott MJ (2002) Metastatic patterns of extremity myxoid liposarcoma and their outcome. J Surg Oncol 80: 8993.

16. Chung PW, Deheshi BM, Ferguson PC (2009) Radiosensitivity translates into excellent local control in extremity myxoid liposarcoma: a comparison with other soft tissue sarcomas. Cancer 115(14): 32563261.

17. Makhlouf HR, Sobin LH (2002) Inflammatory myofibroblastic tumors (inflammatory pseudotumors) of the gastrointestinal tract: how closely are they related to inflammatory fibroid polyps? Hum Pathol 33(3): 307315.

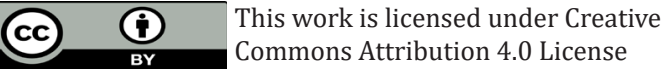

To Submit Your Article Click Here : Submit Article

DOI: $10.32474 /$ RRHOAJ.2018.02.000135

Research and Reviews on
Healthcare: Open Access Journal
Assets of Publishing with us
- Global archiving of articles
- Immediate, unrestricted online access
Rigorous Peer Review Process
- Authors Retain Copyrights

